\title{
Crystal and enzyme environmental effects on ibuprofen and sila-ibuprofen
}

\author{
Florian Kleemiss ${ }^{1}$, Kunihisa Sugimoto ${ }^{2}$, Jens Beckmann ${ }^{1}$, Simon Grabowsky ${ }^{1}$ \\ ${ }^{1}$ University Of Bremen, Bremen, Germany, ${ }^{2}$ JASRI, SPRing-8, Hyogo Prefecture, Sayo, Japan \\ E-mail: florian.kleemiss@uni-bremen.de
}

Ibuprofen is one of the most common and widely used drugs all over the world. It has been classified as one of the most crucial drugs to humankind by the world health organization. Therefore, a deep understanding of the mutual interaction effects between ibuprofen and the involved enzymes (cyclooxygenases) is very important for drug design and pharmaceutical research.

The effect of the crystal environment of a small biologically active ligand in the crystal packing of the pure substance on its electron density has been claimed to be a useful approximation of the polarization in the biological situation.[1] This data is not only relatively easily accessible nowadays, but also based on empirical measurements, while solvation and onion layer models in theoretical calculations might not treat all effects or miss those that are not yet fully understood or parametrized. The derivation of a wavefunction based on the measured electron density, as done in X-ray wavefunction refinement (XWR), [2] is the method which combines theoretical knowledge of the wavefunction with the experimentally observed properties exhibited by the physical crystal. With this technique, it is possible to get better insight into the crystal effect, as well as the similarity and dissimilarity to other environmental influences in solvation and biological systems.

Therefore, ibuprofen (racemic and enantiomerically pure) was crystallized and electron density measurements were carried out at beamline BL02B1, Spring-8, in Japan. The derived X-ray wavefunctions were used to compare properties like electrostatic potential and electron density distribution as well as close contacts in comparison to solvation, enyzme and gas phase situations (QM, QM/MM and MD simulations). These are the properties most crucial for the recognition and binding of an active molecule in the active site of an enzyme.[3]

In addition, and based on the electrostatic rational for ibuprofen, we synthesized sila-ibuprofen (Figure 1) so that the electrostatic properties at the chiral center of the molecule are altered. We obtained both the racemic and enantiomerically pure form and applied the same strategy as for ibuprofen to evaluate whether we can rationally modify and possibly improve the properties of ibuprofen.

[1] Pascard, C. (1995). Acta Cryst. D, 51, 407-417.

[2] Grabowsky, S. et al. (2012). Angewandte Chemie, 51, 6776-6779.

[3] Klebe, G. (1994). J. Mol. Biol, 237, 212-235.

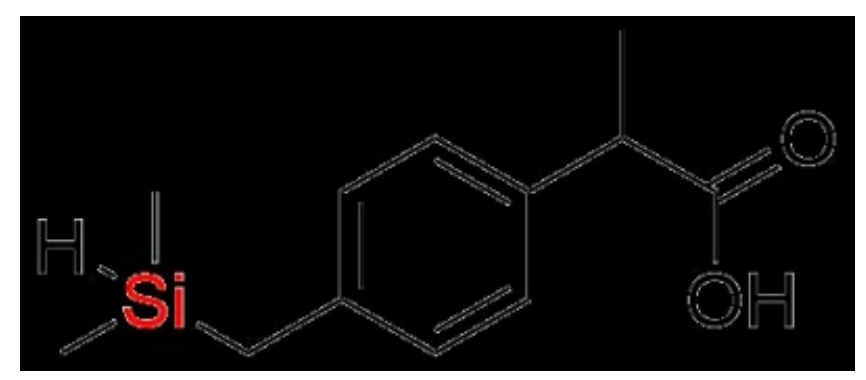

Keywords: Environmental Effects, Crystal-enzyme similarity, Drug simulations 